\title{
Narrativa
}

narrativa

Nuova serie

39 | 2017

Nuove frontiere del Sud

\section{Sulle tracce di Calibano. Subalternità come discorso del mondo-sud}

\section{Mauro Pala}

\section{(2) OpenEdition}

1 Journals

Edizione digitale

URL: https://journals.openedition.org/narrativa/670

ISSN: 2804-1224

\section{Editore}

Presses universitaires de Paris Nanterre

\section{Edizione cartacea}

Data di pubblicazione: 1 décembre 2017

Paginazione: 65-79

ISBN: 978-2-84016-289-6

ISSN: $1166-3243$

\section{Notizia bibliografica digitale}

Mauro Pala, «Sulle tracce di Calibano. Subalternità come discorso del mondo-sud», Narrativa [Online] 39 | 2017, online dal 01 décembre 2021, consultato il 14 janvier 2022. URL: http:// journals.openedition.org/narrativa/670

\section{(c) (1)}

Narrativa est mise à disposition selon les termes de la Licence Creative Commons Attribution 4.0 International. 


\section{Sulle tracce di Calibano. \\ Subalternità come discorso del mondo-sud}

\section{INVISIBILITÀ DEI SUBALTERNI}

Come è possibile, al giorno d'oggi, affrontare una discussione di economia politica e ritrovarsi, nel corso dello stesso scambio di vedute, a trattare di antropologia? Nello stesso contesto, su quali basi un economista individua convincenti analogie fra la crisi europea contemporanea e la storia greca classica ${ }^{1}$ ? In entrambi i casi la risposta ci conduce a due parole chiave del presente: relazione nord-sud e subalternità.

Partendo da una considerazione cursoria del fenomeno, questo testo analizza come la subalternità - concetto imprescindibile dalla sua fenomenologia in varie conformazioni del mondo-Sud - si trasformi, partendo dalla sfera sociologica ed economica, in tematica e insieme strumento di analisi nella critica letteraria e culturale. Subalternità come espressione ambivalente della globalizzazione dunque, proiettata sulla dialettica sociale futuribile in un mondo progressivamente affrancato dagli stati nazionali e dai relativi apparati obsolescenti, ma anche ipotesi comunitaria che, sullo sfondo della galassia postcoloniale, rielabora la memoria in vista di forme rizomatiche di aggregazione sovranazionali. Un tale complesso presuppone "la centralità della costruzione di nuovi spazi prodotti dal capitalismo globale [partendo] da studi della

1. Per illustrare la debolezza negoziale di Keynes rispetto alla delegazione statunitense in occasione della conferenza di Bretton Woods, Varoufakis rievoca da La guerra del Peloponneso di Tucidide l'arroganza dei potenti generali ateniesi, i quali spiegavano agli abitanti dell'isoletta di Melo - nemica di Atene - che "i diritti" valgono solo "fra chi ha uguali poteri" per cui loro avrebbero fatto "quello che più gli piaceva". "Perché i forti fanno quello che gli aggrada e i deboli sono destinati a soffrire" (VArOUFAKIS, Yanis, I deboli sono destinati a soffrire?, Milano, La nave di Teseo, 2016, p. 46). 
rappresentazione letteraria dello spazio" ${ }^{2}$ la cui comprensione richiede competenze interdisciplinari.

Partendo da queste premesse, la discussione che si è ipotizzata non potrebbe che essere multidisciplinare. Per diagnosticare una condizione subalterna il confronto potrebbe prendere le mosse dall'opinione di Giovanni Arrighi che già nel 1999 avanzava seri dubbi sulla tenuta di stati e imprese chiedendosi se non stiamo assistendo ad una "caduta libera dei gruppi subordinati", per continuare con Thomas Piketty, il quale nel 2013 apre provocatoriamente il suo importante studio sul capitale nel ventunesimo secolo con l'articolo 1 della Dichiarazione dei diritti dell'uomo e del cittadino del 1789: "le distinzioni sociali non possono fondarsi che sull'utilità comune"; e passare poi meticolosamente in rassegna gli sviluppi odierni della distribuzione del reddito, prendendo in considerazione fattori decisivi come l'istruzione ${ }^{5}$ e le politiche pubbliche seguite negli anni dai principali stati occidentali, per approdare a un corposo capitolo sulla "Disuguaglianza mondiale dei patrimoni nel XXI secolo"s. Parallelamente a come è cambiata nei decenni la concezione della ricchezza, è mutata anche la nozione di coloro che subiscono la diseguaglianza, ovvero quei gruppi o individui identificati oggi generalmente come subalterni, in base alla cui diffusione e natura dipende la ripartizione fra mondo Nord e mondo Sud. La stessa antinomia fra questi due poli va considerata sia in una prospettiva macrogeografica - in cui effettivamente le terre dell'emisfero settentrionale coincidono con gli stati più ricchi del pianeta - sia in un'ottica locale e regionale, in cui, ad esempio, i Länder settentrionali della Repubblica Federale costituiscono il "sud" socioeconomico della pur ricca Germania. Quest'ultima tendenza a macchia di leopardo va accentuandosi via via che l'analisi si fa più specifica e situazionista, tanto che, soprattutto in Asia, anche il neoliberismo - generalmente associato a una politica economica asservita al capitale finanziario e ostile allo stato - si configura invece come uno strumento nelle mani dello stato per calibrare il consenso e, in estrema istanza, "per l'esercizio di politiche autoritarie".

2. Benvenuti, Giuliana, Ceserani, Remo, La letteratura nell'era globale, Bologna, Il Mulino, 2012, p. 108.

3. Arrighi, Giovanni, Silver, Beverly J., Caos e governo del mondo, Milano, Bruno Mondadori, 2003, p.4.

4. PIKeTty, Thomas, Il capitale nel XXI secolo, Milano, Bompiani, 2014, p. 9.

5. "lo stato del sistema formativo e lo stato della tecnologia svolgono un ruolo essenziale e influiscono non poco sui rapporti di forza tra i diversi gruppi sociali interessati" (Ibid., p. 467).

6. Ibid., p. 665 e ss.

7. Ong, Aihwa, Neoliberalismo come eccezione. Cittadinanza e sovranità in mutazione, Firenze, La Casa Usher, 2013, p. 10. 
Sullo sfondo di una governamentalità foucaultiana sempre più diffusa e differenziata ${ }^{8}$ così come il quadro giuridico globale che ne risulta, economisti, studiosi di scienza politica, sociologi concordano sul fatto che la disuguaglianza sociale, oggigiorno ridotta a istanza secondaria o assente nell'agenda dei principali gruppi politici che ambiscono a governarci, è divenuta strutturale nel sistema mondiale delineatosi dopo il 1971, col venir meno dell'azione concertata a Bretton Woods e la progressiva finanziarizzazione del capitalismo: in quella "società del rischio" che contraddistingue la seconda modernità il problema delle diseguaglianze ha paradossalmente

perso la sua esplosività sociale [per riemergere] in nuovi contesti e varianti (discussione sulla nuova povertà, lotta per i diritti delle donne, iniziative civiche contro le centrali nucleari [...]). Ma se prendiamo la discussione pubblica e politica come indice dello sviluppo reale, allora la conclusione che si impone è la seguente: oggi nella Repubblica Federale, sebbene le vecchie diseguaglianze non siano scomparse e ne sorgano delle nuove, viviamo in condizioni che sono al di là della società di classe, e in cui l'immagine della società di classe è mantenuta in vita solo per mancanza di un'alternativa migliore?.

Come risulta evidente, questa breve rassegna sul contrasto fra mondo Nord e mondo Sud rischia di arenarsi davanti alla mancata visibilità dei subalterni, frutto, almeno in parte, della stessa ipertrofia di stimoli e dati (solo tangenziali, mai sostanziali rispetto al tema) messi in campo dagli studiosi finora citati. Dobbiamo dunque rassegnarci all'eclisse dei subalterni nel mondo globalizzato, dichiararne l'indecifrabilità nonostante il loro ruolo rilevante nel dibattito in corso su cittadinanza ${ }^{10}$ e nuove pratiche identitarie nazionali?

La soluzione, già prospettata nel titolo, è quella di una trattazione antropologica in senso lato, ovvero discorsiva, in cui, pur dando per acquisita la sovrapposizione fra il testuale e l'ideologico, teniamo a mente che "discorso non è solo

8. Adotto la definizione di Sandro Chignola per "governamentalità": "Ciò che emerge da questa riformulazione dell'analitica del potere foucaultiana mi sembra essere, piuttosto, una fuoruscita definitiva dall'identificazione di politico e statuale disposta dal moderno concetto di sovranità" (Chignola, Sandro, Foucault oltre Foucault. Una politica della filosofia, Roma, Deriveapprodi, 2014, p. 72).

9. BECK, Ulrich, La società del rischio. Verso una seconda modernità, Roma, Carocci, 2005, p. 117.

10. Cfr. Chatterjee, Partha, The Nation and its Fragments. Colonial and Postcolonial Histories, Princeton, Princeton U.P., 1993, ID., The Politics of the Governed, New York, Columbia University Press, 2004; Chaturvedi, Vinayak (ed.), Mapping Subaltern Studies and the Postcolonial, London, Verso, 2012. 
un altro modo per chiamare le rappresentazioni. L'analisi del discorso, invece, richiede lo studio delle condizioni sociali e storiche in cui vengono generate le rappresentazioni" ${ }^{11}$. E per riattivare una storia dei subalterni e del sud farò riferimento, senza alcuna pretesa di esaustività, a una narrazione che sappia far aggio sulle acquisizioni della decostruzione - come peraltro molti degli autori succitati, ancorché attivi in altri campi - e, allo stesso tempo, adottare, con l'affermarsi della "grande triade Barthes, Foucault Bachtin (...) un'idea di lettera-

tura come critica culturale, come critica del rapporto fra parole e cose, fra linguaggio e potere": dove, citando Calvino, la letteratura sappia "porsi tra la forma della fiamma e quella del cristallo, tra le parole quotidiane che dicono troppo e quelle che dicono troppo poco" ${ }^{12}$. Supporto letterario per un percorso a ritroso, verso l'origine della questione subalterna, attraverso le voci che hanno agitato quella questione.

\section{Genealogia dei subalterni}

Caliban: Hast thou not dropp'd from heaven? Stephano: Out o'the moon, I do assure thee: I was The man I'the moon when time was.

Caliban: I have seen thee in her, and I do adore thee: My mistress show'd me thee, and thy dog, and thy bush Stephano: Come, swear to that, kiss the book: I will Furnish it anon with new contents: swear.

Calibano: ma non sei caduto dal cielo?

Stefano: sono caduto dalla luna, te lo assicuro; una volta ero l'uomo della luna. Caliban: ti ho visto, lassù.

Sempre, la mia padrona, Ti indicava a me, Col cane e la fascina.

E io ti adoro.

Stefano: avanti, giura - bacia il messale! Tra poco ce ne metto ancora - giura ${ }^{13}$.

11. Loomba, Ania, Colonialismo/postcolonialismo, Roma, Meltemi, 2000, pp. 105-106.

12. Sobrero, Alberto, Il cristallo e la fiamma. Antropologia fra scienza e letteratura, Roma, Carocci, 2011, p. 38.

13. Shakespeare, William, La tempesta, Milano, Garzanti, 1984, p. 97 (atto secondo, scena seconda). 
Assai prima della hegeliana dialettica servo-padrone Shakespeare rappresenta da par suo le gerarchie sociali nelle loro dinamiche e contraddizioni, ulteriormente amplificate dal momento storico in cui culture differenti si fronteggiano in una situazione di colonizzazione incipiente. Si tratta della seconda scena del secondo atto della Tempesta, allorché Calibano incontra i due fool, Stefano e Trinculo, che lo fanno bere fino a che, ubriaco, inneggerà sguaiatamente al suo affrancamento da Prospero. Per quanto il registro della scena sia quello della satira per effetto della degradatio, il messaggio shakespiriano è molto chiaro ed efficace: Calibano e Stefano pasticciano, si ripetono, risultano comici e non riescono veramente a interloquire perché il loro spazio d'azione è l'Esterno, "quello spazio ritualmente devoluto, cioè consegnato e assegnato a tutto il personale non-tragico, una sorta di ghetto alla rovescia" 14 popolato da informatori, tirapiedi, schiavi e servi di varia natura, accomunati dall'incapacità di dominare sia il codice espressivo in uso al tempo che lo spazio su cui questo codice opera, ed essere dunque confinati nell'angolo della chiacchiera licenziosa, in cui Shakespeare però indugia volentieri ${ }^{15}$, sapendo che è funzionale alla preparazione dell'evento tragico.

Costretti ai margini dell'azione, nei loro discorsi bizzarri Calibano e Stefano fanno ricorso a triti luoghi comuni: la religione appiattita nell'atto di baciare il messale, la cosmogonia popolare con l'uomo della luna, elementi di una cultura d'accatto che nega la celebre affermazione di Bachtin secondo cui "L'essere dell'uomo è una comunicazione profonda. Essere significa comunicare. Essere significa essere per l'altro e, attraverso l'altro, per sé"16. Per il Bardo i perdenti sono coloro che non sanno leggere i segni del mondo, e, siano essi sovrani vittime delle proprie ossessioni, come Macbeth o Lear, o semplici diseredati, li accomuna il limite di fermarsi alle apparenze, di non sapersi emancipare da un particulare che ottunde le loro capacità di comprensione della complessità.

Il grande limite della colonizzazione occidentale, evidentemente, risiede nella stessa tendenza omologante, studiata in modo esemplare da Todorov nella figura di Colombo ermeneuta

14. BArthes, Roland, “L'uomo raciniano”, in ID., Saggi critici, Torino, Einaudi, 1972, p. 144.

15. "For wantonness in strength is a mark of the absolutely mature Shakespeare, as perhaps of all poets; it arises from a confidence that one has created the context in which one can be understood" (Kermode, Frank, Shakespeare, Spencer Donne, London, Routledge and Kegan, 1971, p. 162).

16. 'Todorov, 'Tzvetan, La conquista dell'America. Ilproblema dell'Altro, Torino, Einaudi, 1984, p. VIII. 
che traduce [la differenza] immediatamente in termini di superiorità (nel suo caso, com'è ovvio, sono gli indiani ad essere considerati inferiori): si nega l'esistenza di una sostanza umana realmente altra, che possa non consistere semplicemente in un grado inferiore, e imperfetto, di ciò che noi siamo. Queste due figure dell'alterità si fondano entrambe sull'egocentrismo, sull'identificazione dei propri valori con i valori in generale, del proprio io con l'universo: sulla convinzione che il mondo è uno ${ }^{17}$.

Tali presupposti si manifestano appieno nel colonizzatore che, sulla scia di Colombo, "crede che i suoi valori siano i valori e ciò che gli è sufficiente"18; avvalorando "l'analogia tra la rappresentazione cartografica e il mercato che ne è la sua messa in opera" 19 una schiera di zelanti operatori contribuiranno nel corso dell'espansione coloniale europea a coniugare in senso geografico l'assolutizzazione dei valori tipica dell'Illuminismo, esemplificata da Voltaire quando traduce in termini diacronici i presupposti dell'Esprit des lois di Montesquieu, assimilando, fra le altre terre interessate dal fenomeno, il Mezzogiorno d'Europa all'Oriente: non si tratta soltanto di collocare l'Europa occidentale in una dimensione temporale diversa e più consona al suo status materiale, ma di riaffermare, con un'immagine prometeica dalle forti tinte romantiche, lo iato che separa il Nord Europa dalla stasi e dal clima debilitante cui invece sottostanno l'Oriente e il Sud europeo: la conferma di una supremazia, culturale e tecnologica insieme, arriva anche dalla letteratura, che celebra la ferrea volontà collettiva francese di superare i vincoli dello stato di natura ${ }^{20}$.

L'eurocentrismo nelle sue varianti ermeneutiche sullo sfondo universalista costituisce uno "schema, una rappresentazione astratta, prodotta attraverso un lavoro specifico di costruzione, ed in grado di offrire, proprio come una carta geografica, una visione dall'alto, un punto di osservazione sull'insieme dei punti, a partire dai quali attori sociali gettano il loro sguardo sul mondo sociale" ${ }^{21}$. A questi criteri si rifà anche la sociologia comtiana per giudicare e classificare la dicotomia fra Nord e Sud del mondo: una divulgazione che lucra sul prestigio di tutto ciò che è scientifico, ma che spesso ricicla e conferisce prestigio a nozioni

\section{Ibid., p. 51.} p. 6.

18. ID., Noi e gli altri. La riflessione francese sulla diversità umana, Torino, Einaudi, 1991,

19. Farinelli, Franco, La crisi della ragione cartografica, Torino, Einaudi, 2009, p. 28. 2007.

20. Cfr. Dainotтo, Roberto, Europe (In Theory), Durham, Duke University Press,

21. Bourdieu, Pierre, La distinzione. Critica sociale del gusto, Bologna, Il Mulino, 1983, p. 173. 
di senso comune ${ }^{22}$. E così in Occidente l'asse Nord-Sud compendia una conoscenza superficiale che però, grazie ai semplicistici schemi di raffronto binario cui si affida - Kultur-Zivilisation, evoluto-selvaggio - ha una facile presa sulle masse, oltre che successo nel suggellare l'interdipendenza fra "produzione di conoscenza e strategie di rappresentazione"23. Porsi sulle tracce di Calibano significa, come già aveva intuito Barthes, non tanto analizzare le condizioni del suo essere (che non esiste in sé) come subalterno, quanto la possibilità (o volontà) della sua rappresentazione, riallacciandosi alla brillante apertura di Orientalismo con la citazione dal Diciotto Brumaio di Louis Bonaparte: "Non possono rappresentare sé stessi; devono essere rappresentati” ${ }^{24}$ dei subalterni. Evidentemente, nel momento in cui il Sud d'Italia diventa palcoscenico di una proiezione orientalista ${ }^{25}$, le logiche della subalternità realmente si applicano al mondo-Sud, essendo ritenute le più opportune per quella tipologia di luogo, secondo la logica missionaria gesuitica che pone sullo stesso piano l'Italia meridionale e le Indias de por acá. Metafora non occasionale - come osserva Ernesto de Martino - di un'analogia effettiva "fra il nuovo mondo da guadagnare alla civiltà cristiana e le condizioni culturali di larghe zone del vicereame di Napoli” 26 . Considerati questi esiti subalterni, non stupisce che critici postcoloniali ed esponenti del pensiero meridionale contemporaneo ${ }^{27}$ rifiutino quel modello hegeliano di storiografia considerato manifesto di una certa modernità ${ }^{28}$.

Lo spunto per una nuova valutazione di questo appiattimento del mondosud su quello che è divenuto intanto un paradigma della subalternità arriva ancora da Edward Said del 1988, col saggio "Rappresentare i colonizzati.

22. Cfr. Connell, Raewyn, Southern Theory, Cambridge, Polity, 2007.

23. Loomba, Ania, Colonialismo/postcolonialismo, cit., p. 106.

24. SAID, Edward, Orientalismo. L'immagine europea dell'Oriente, Milano, Feltrinelli, 2014 [1978], p. 11.

25. Una tesi centrale in SchneIDER, Jane (a cura di), Italy's Southern Question. Orientalism in One Country, Oxford, Berg, 1988.

26. De Martino, Ernesto, La terra del rimorso. Contributo a una storia religiosa del Sud, Milano, Il Saggiatore, 1961, p. 23 cit. in MoE, Nelson, Un paradiso abitato da diavoli, Napoli, l'ancora del mediterraneo, 2004, p. 59.

27. "I pericoli che l'homo currens produce non nascono dalla velocità, ma dalla sua assolutizzazione, dall'identificazione di essa con il progresso" (Cassano, Franco, Il pensiero meridiano, Bari, Laterza, 2005, p. XVI).

28. Una posizione condivisa, fra gli altri, da Chambers, Iain, Le molte voci del Mediterraneo, Milano, Raffaello Cortina, 2013, la succitata Jane Schneider (1988) e CHAKRABarty, Dipesh, Provincializare l'Europa, Roma, Meltemi, 2004, in particolare il capitolo "Storie delle minoranze, passati subalterni", p. 135 e ss. 
L'antropologia e i suoi interlocutori'" ${ }^{29}$. Il critico palestinese rileva come già alla fine del Novecento la crisi della rappresentazione in Occidente sia un dato acquisito, tanto che le ricadute del fenomeno vengono metabolizzate anche dalla critica letteraria: in questo clima di incertezza epistemologica le grandi opere di Abrams o di Auerbach costituiscono gli ultimi esempi di un'analisi in cui la parola ancora appare quel "medium trasparente che consentiva all'Essere di manifestarsi" 30 .

Secondo Said, una simile opacizzazione del linguaggio, graduale ma irreversibile, è stata un problema per quanti, da Marx a Nietzsche a Freud, hanno cercato di inquadrare le linee evolutive della modernità, ostacolati in questo anche dalla concorrenza di altri fattori strutturali alla comunicazione, come l'inconscio, la razza, le convenzioni legate al gender, ovvero tutti quegli elementi che costituiscono il substrato discorsivo del linguaggio stesso. Oggi dunque, sullo sfondo di un contesto ermeneutico nel quale le nozioni di soggetto o di autore hanno progressivamente perso senso, Said si chiede come sia possibile porsi rispetto a un fenomeno come la colonizzazione, intesa in un'accezione sempre più ampia, in cui rientra evidentemente anche la discriminante NordSud. "La categoria di colonizzato ha sensibilmente esteso il suo campo semantico, fino ad includere la condizione delle donne, quella di tutte le classi sfruttate e oppresse, le minoranze nazionali" incalza Said, e "colonizzato significa oggi essere cose potenzialmente anche molto diverse, in posti diversi e in epoche diverse, ma sempre inferiori" ${ }^{31}$. Ciò che il pensatore palestinese sollecita in primo luogo è una definizione di colonizzazione, ovvero la possibilità di poterla raccontare, di includerla in una sintassi "di un certo modo di esprimere la nostra comprensione del mondo" 32. L'ansia di narrazione e, allo stesso tempo, il desiderio di estendere l'area semantica del lemma "colonizzato" da parte di Said non può non richiamare il celeberrimo Can the Subaltern Speak di Gayatri Spivak, pubblicato lo stesso anno ${ }^{33}$.

29. SAID, Edward, "Rappresentare i colonizzati. L'antropologia e i suoi interlocutori”, in ID., Nel segno dell'esilio. Riflessioni, letture e altri saggi, Milano, Feltrinelli, 2008, pp. 340 e ss.

30. Ibid., p. 340.

31. Ibid., p. 343.

32. Brooks, Peter, Trame. Intenzionalità e progetto nel discorso narrativo, Torino, Einaudi, 2004, p. 7.

33. SpIvak, Gayatri Chakravorty, "Can the Subaltern Speak?” in Nelson, Cary, Grossberg, Lawrence (a cura di), Marxism and the Interpretation of Culture, Chicago, University of Illinois Press, 1988, pp. 271-313. 
Se è pressoché impossibile sintetizzare le varie problematiche affrontate in un saggio che ha avuto una eco globale e duratura, si può osservare una critica ricorrente a Marx per ciò che concerne il capitalismo globale e l'alleanza fra le nazioni che ha sostenuto quelle forme di sfruttamento - fra cui, ovviamente, la colonizzazione - che al capitalismo planetario vanno ricondotte; secondo la studiosa di origine bengalese, la critica marxiana non è errata in sé, ma si sviluppa su una scala macroeconomica talmente elevata da perdere di vista la dimensione intersoggettiva, che richiede piuttosto un vaglio accurato delle ideologie in gioco. È infatti a livello di microanalisi che si attuano processi di formazione del soggetto, spesso imprevedibili eppure cruciali nello smentire le macro tendenze ${ }^{34}$.

\section{Prospettive sui/ Dei subalterni}

In questa fase, caratterizzata dalla ricerca di un soggetto storico - ovvero di una agency - svincolato/a dall'eredità determinista dell'idealismo, ma che, come attore sociale, riconosca, a differenza di quanto sostiene Deleuze, l'esistenza e il ruolo delle forme di rappresentazione, entra in scena Gramsci con la sua analisi dettagliata della Questione Meridionale. Anche per ciò che concerne l'apprezzamento del pensatore sardo Said e Spivak mostrano sorprendenti affinita ${ }^{35}$ : lo studioso palestinese ritiene

la consapevolezza geografica di Gramsci particolarmente adatta alla critica letteraria del tardo XX secolo, chiamata a confrontarsi con formazioni ed esperienze distinte e separate come la storia delle donne, la cultura popolare, le vicende postcoloniali e il postmoderno: tutte tematiche che difficilmente possono essere assimilate fra loro, e che non si lasciano assoggettare o inquadrare in uno schema di corrispondenze omnicomprensivo ${ }^{36}$.

34. "the relationship between global capitalism (exploitation in economics) and nation-states alliances (domination in geopolitics) is so macrological that it cannot account for the micrological texture of power. To move toward such an accounting one must move toward theories of ideology - of subject formations that micrologically and often erratically operate the interests that congeal the macrologies" (Ibid., p. 279).

35. Nella rispettiva relazione con gli scritti di Gramsci occorre tener presente che già negli anni Ottanta Said aveva accesso all'edizione critica dei Quaderni, mentre Spivak utilizzava la Selections from the Prison Notebooks curata da Quintin Hoare e Geoffrey Nowell Smith (London, Lawrence \& Wishart, 1971), in cui gli scritti sui subalterni sono collocati all'interno della terza sezione "Notes on Italian History" che non ha un riscontro nell'edizione critica.

36. SAID, Edward, "Storia, letteratura e geografia", in ID., Nel segno dell'esilio, cit., p. 510 . 
Singolare il giudizio su Gramsci della Spivak ${ }^{37}$, la quale osserva come il prigioniero politico, costretto dalla censura carceraria, usi il termine "subalterno" al posto di "proletario", facendo confluire in quella parola tutto ciò che non rientra in una precisa analisi di classe: proprio questa assenza di rigore teorico è l'elemento che la Spivak maggiormente apprezza. Anche se la filologia gramsciana recente ha messo in luce una sistematica rete di corrispondenze in cui i subalterni si collocano, l'opinione della comparatista bengalese segnala l'intenzione di servirsi dei subalterni come un mezzo per rinnovare l'intero apparato teorico radical.

Riguardo alla genesi del lemma in ambito speculativo, negli scritti pre-carcerari Gramsci utilizza in diverse occasioni la qualifica, corrente nella gerarchia militare, di "subalterno" - termine immediatamente comprensibile in tutta Europa all'indomani del primo conflitto mondiale, parola che progressivamente assume un significato più ampio, metaforico; poi, nel corso della riflessione carceraria, una vera e propria teoria originale sui diversi aspetti delle relazioni culturali e politiche fra dominanti e subalterni prende forma. Sintesi di questa rielaborazione è il Quaderno 25 - quaderno speciale intitolato "ai margini della storia" - che Gramsci assembla in modo asistematico nel 1934 partendo dalle note pregresse; essendo l'unico quaderno speciale su un tema che non figura nella lista degli argomenti principali programmati nel Quaderno 1, si tratta di una formulazione innovativa e aperta come un vero work in progress: caratteristiche che si riflettono nei criteri metodologici da adottare nei riguardi dei subalterni ${ }^{38}$.

La storia dei gruppi subalterni è necessariamente disgregata ed episodica. Ł̀ indubbio che nell'attività storica di questi gruppi c'è la tendenza all'unificazione sia pure per periodi provvisori, ma questa tendenza è continuamente spezzata dall'iniziativa dei gruppi dominanti, e pertanto può essere dimostrata solo a ciclo storico compiuto, se esso si conclude con un successo. I gruppi subalterni subiscono sempre l'iniziativa dei gruppi dominanti, anche quando si ribellano e insorgono. Solo la vittoria "permanente" spezza, e non immediatamente, la subordinazione. In realtà, anche quando paiono trionfanti, i gruppi subalterni

37. “the word 'subaltern' was used under censorship by Gramsci: he called Marxism 'monism' and was obliged to call the proletarian 'subaltern'. That word, used under duress, has been transformed into the description of everything that doesn't fall under strict class analysis. I like that, because it has no theoretical rigor" (SPIVAK, Gayatri, "Gayatri Spivak and the Politics of the Subaltern", in Socialist Review, n. 20, lugliosettembre, 1990, p. 86).

38. Gramsci, Antonio, I Quaderni del carcere, Torino, Einaudi, 1975, Q 25, S 2, p. 2283. 
sono solo in uno stato di difesa allarmata [...] Ogni traccia di iniziativa autonoma da parte dei gruppi subalterni dovrebbe perciò essere di valore inestimabile per lo storico integrale; da ciò risulta che una tale storia non può che essere trattata che per monografie e che ogni monografia domanda un cumulo molto grande di materiali spesso difficili da raccogliere ${ }^{39}$.

Ponendo l'accento sulla capacità di scoprire nella traccia l'impronta del tutto, Gramsci implicitamente si candida al ruolo di "storico integrale" in grado di assemblare in un insieme coerente gli indizi di una possibile iniziativa autonoma da parte di gruppi o anche individui isolati. Nella citazione risaltano le linee portanti dell'esegesi storica gramsciana: qualsiasi discorso sulla subalternità deve avere un carattere contingente ed empirico, in quanto l'analisi dei fenomeni subalterni è particolarmente delicata, e richiede, prima ancora di raccogliere i dati, una revisione, da parte dello studioso, delle categorie e degli strumenti ermeneutici di cui si è servito fino a quel momento. In tale prospettiva, il sistema adottato da Gramsci per comprendere e, insieme, raccontare il Mezzogiorno visto come repertorio di varie subalternità è induttivo, implica cioè un capovolgimento di quel metodo deduttivo, di matrice illuminista, che dominava allora le scienze sociali e allignava anche nel marxismo schematizzato, deterministico e divulgativo nell'Unione Sovietica di Bucharin; per molti versi l'ottica gramsciana costituisce una reazione consapevole alle tentazioni universaliste presenti nel marxismo successivo a Engels e nel progetto fallimentare della Seconda Internazionale.

Nell'ermeneutica eterodossa del pensatore sardo c'è la chiave per capire quella ricerca storica "per monografie". Azzardo che questo modo di procedere possa prevenire anche l'opacizzazione del linguaggio cui si riferiva Said: l'affievolirsi dell'autorità del narratore dopo la grande stagione strutturalista trova nell'approccio genealogico-filologico auspicato da Gramsci sia un rimedio euristico che una risposta tangibile all'evidente iato nella storiografia post Hayden White - ma anche nella narrativa modernista - fra le fonti storiche e la loro rielaborazione, fra $\mathrm{i}$ fatti e la possibilità di narrarli. Come sostiene Carlo Ginzburg, "tra testimonianze, sia narrative sia non narrative, e realtà testimoniata esiste un rapporto che dev'essere analizzato di volta in volta" 40 .

Trattando dei subalterni il ricorso a una verifica costante delle condizioni della narrazione è non solo opportuno per la natura "rapsodica" ed effimera del tema, ma anche in relazione a come la questione viene studiata: come già a

39. Ibid., pp. 2283-2284.

40. Ginzburg, Carlo, Il filo e le tracce. Vero falso finto, Milano, Feltrinelli, 2006, p. 8. 
suo tempo diagnosticato da Bloch, "ciò che ci dice il testo non costituisce più l'oggetto preferito della nostra attenzione" 41 e quindi la logica della rivelazione imperniata su un autore/auctoritas è venuta meno da tempo, come già sottolinea Said a proposito della rappresentazione dei colonizzati, e con la modernità si afferma una radicale "sfiducia nella possibilità di evocare il passato" - con buona pace di Ranke e della sua storiografia - "come un tutto compiuto. Al suo posto comincia ad affiorare la consapevolezza che la nostra conoscenza del passato è inevitabilmente incerta, discontinua, lacunosa: basata su una massa di frammenti e di rovine" 42 .

L'inflessione archeologica-antropologica dell'analisi gramsciana segna il passaggio a uno scrutinio puntiglioso del contesto (piuttosto che del testo, considerato nella storiografia tradizionale come fonte univoca, o almeno preminente), un'indagine sulle condizioni che hanno reso possibile un evento, anche un episodio improbabile come l'ascesa del movimento millenaristico di Davide Lazzaretti presso il monte Amiata, nella fase immediatamente postunitaria. Ritenuto un "folle" da Cesare Lombroso e un pericoloso sovversivo dalle autorità statali che l'avrebbero infine fucilato, il visionario toscano raccoglie l'eredità delle jacquerie antifeudali ma, soprattutto, per ciò che riguarda il nostro tema, rappresenta la possibilità effettiva di un gruppo subalterno di organizzarsi fino a costituirsi in uno stato autonomo ${ }^{43}$. Senza dover attendere che le condizioni economiche circostanti fossero mature, in un ambiente rurale e non progredito (nel senso di industrializzato), e facendo riferimento a una visione del mondo - Foucault parlerebbe di "episteme" - radicalmente diversa da quella egemone, borghese. Non solo qui Gramsci ritiene possibile l'emancipazione dei subalterni, ma fa il primo passo in questo senso rivalutando la controversa sfera del folklore, contrariamente a quello che fecero generazioni di intellettuali italiani "cosmopoliti" che confinarono generazioni di subalterni nel ghetto degli umili ${ }^{44}$.

Su questi aspetti si registra l'ideale convergenza fra la trattazione gramsciana e l'auspicio da parte di Said di un mutamento di indirizzo per l'antropologia. Basta con una disciplina fossilizzata "sulla rappresentazione di realtà non occidentali considerate primitive o meno evolute", ciò che occorre invece è un'antropologia $50-51$.

41. Bloch, Marc, Apologia della storia o Mestiere di storico, Torino, Einaudi, 1998, pp.

42. GinzBurg, Carlo, Il filo e le tracce. Vero falso finto, cit., p. 38.

43. Cfr. Green, Marcus, "Sul concetto gramsciano di subalterno", in VACCA, Giuseppe, SCHIRru, Giancarlo (a cura di), Studi gramsciani nel mondo 2000-2005, Bologna, Il Mulino, 2007, p. 217.

44. Gramsci, Antonio, I Quaderni del carcere, cit., Q 21, \$3, p. 2112. 
più letteraria, "o meglio teorico-letteraria per stile e consapevolezza: un'antropologia cioè che dedichi più tempo alla riflessione sulla testualità del proprio sapere e meno alla discendenza matrilineare" ${ }^{45}$. Un "esaurimento di paradigma" secondo Said, quello dell'antropologia tradizionale, che consiglia di puntare sull'unica "possibile alternativa in una pratica fondata proprio sulla pratica, e rafforzata da idee e concetti come quelli di egemonia, di riproduzione sociale e di ideologia, tutti mutuati da autori - Gramsci, Raymond Williams, Alain Touraine e Pierre Bourdieu - che antropologi di certo non sono" 4 .

Una proposta coerente con la traiettoria gramsciana in cui l'immanenza si qualifica come pratica reale, un sapere dell'azione concreta, dei bisogni concreti, delle necessità concrete ${ }^{47}$. La stessa fenomenologia della subalternità rientra in una prassi (e sottolineo praxis) come concezione immanente, sia degli eventi in questione e della storia in generale, contrariamente alle soluzioni idealiste che virano verso il materialismo, adottate, fra gli altri, da Lukacs in Storia e coscienza di classe e da Korsch ${ }^{48}$ in Marxismo e Filosofia.

Mentre nella fase pre-carceraria Gramsci fa ancora ricorso a categorie idealistiche, all'atto della redazione dei Quaderni adotta una concezione delle relazioni sociali come "organizzazione di rapporti di conoscenza" ${ }^{49}$ : sotteso a questo passaggio "è il concetto stesso di cultura che nei Quaderni si presenta come essenzialmente nuovo. Questa novità sta nella filosofia della praxis, e nella sua premessa, l'unità di teoria e pratica, di filosofia e politica" ${ }^{\text {50. }}$. Ma con la filosofia della prassi la necessità di una verifica rispetto alle condizioni contingenti diviene ancora più pressante, perché la rinuncia a una legge generale cui fare ricorso nasce dalla percezione che la sfera sociale non è mai stabile $e^{51}$, ma risente di

45. SAID, Edward, Nel segno dell'esilio. Riflessioni, letture e altri saggi, cit., p. 343.

46. Ibid., p. 345.

47. Si tratta di passaggi che possiamo riscontrare nei Q 4 (p. 432) e 5 (p. 657).

48. Tale concezione immanente è un'innovazione fondamentale, una scelta grazie alla quale Gramsci si candida a riformatore di un marxismo che "ha dovuto allearsi con tendenze estranee per combattere i residui del mondo pre-capitalistico nelle masse popolari" (cfr. Q 4, p. 422).

49. Frosini, Fabio, La religione dell'uomo moderno. Politica e verità nei Quaderni del carcere di Antonio Gramsci, Roma, Carocci, 2010, p. 57.

50. Ibid., p. 58.

51. "La filosofia della praxis è nata sul terreno del massimo sviluppo della cultura della prima metà del secolo XIX, cultura rappresentata dalla filosofia classica tedesca, dall'economia classica inglese, e dalla letteratura e pratica politica francese [...] La filosofia della praxis ha elaborato sinteticamente i tre movimenti, cioè l'intera cultura dell'epoca e nella sintesi nuova, in qualsiasi momento la si esamini, momento teorico, economico, politico, si ritrova come momento preparatorio ognuno dei tre 
spinte centrifughe e centripete, sintomi di una contesa egemonica in atto. Le categorie di giudizio si definiscono in corso d'opera e la subalternità, come l'egemonia, è una condizione instabile, in quanto discende da una relazione in atto.

Una storia, quella dei subalterni in Gramsci, che, così come in James Clifford, "migra tra prospettive locali e globali, in una continua ricontestualizzazione del suo oggetto" 52 . Una congiuntura propizia per la decostruzione che Spivak opera nei confronti del marxismo, rivendicando i limiti della prospettiva di Marx ${ }^{53}$ nella sua esclusione di una dimensione gender della sua analisi/denuncia, aggravata dalla concomitanza fra pratiche coloniali e tradizioni sessiste perpetrate in un territorio inequivocabilmente parte del mondo-sud ${ }^{54}$. Ma la soluzione che Spivak adotta è quella di bruciare i ponti (teorici) alle spalle, per evitare di incorrere nella trasgressione di un nuovo ismo, nel corso dell'inevitabile involuzione prescrittiva che anche tale filone di pensiero subirebbe. Anche questo rifiuto simbolico delle origini rientra nel discorso della subalternità, praticato sotto forma di decostruzione militante:

la decostruzione spivakiana diventa, specularmente, un gesto estremo di militanza politica nel programmatico rifiuto [da parte di Spivak] di recuperare le origini, prendendo così le distanze dalle seduzioni essenzializzanti dell'antropologia occidentale, e invece cercando di collocare le pratiche culturali e politiche dei gruppi subalterni sull'orizzonte mobile della testualità, intesa nella sua più ampia accezione di processo prodotto, e produttivo, di differenze $\mathrm{e}^{55}$.

Avendo a che fare con una relazione instabile - la subalternità - in cui anche la mappatura demografica e macroeconomica dei poli Nord e Sud subisce continue variazioni, la decostruzione corre il rischio di risultare inefficace intervenendo in ritardo su situazioni che si sono già risolte, alterando irreversibilmente gli stessi

movimenti. [...] E il momento sintetico unitario mi pare da identificare nel nuovo concetto di immanenza, che dalla sua forma speculativa [...] è stato tradotto in forma storicistica” (GramscI, Antonio, I Quaderni del carcere, cit., Q 10, \9, pp.1246-1247, cit., in Crehan, Kate, Gramsci, cultura e antropologia, Lecce, Argo, 2010, p. 95).

52. ClifFORD, James, I frutti puri impazziscono. Etnografia, letteratura e arte nel secolo XX, Torino, Bollati Boringhieri, 2004, p. 22.

53. "Tanto nell'area economica (il capitalista) quanto in quella politica (l'agente storico-mondiale), Marx è obbligato a costruire i modelli di un discorso diviso e dislocato, le cui parti non sono continue e coerenti fra loro" (SPIVAK, Gayatri Chakravorti, Critica della ragione postcoloniale, Roma, Meltemi, 2004, p. 271).

54. Il riferimento è, ancora una volta a SPIVAK, Gayatri Chakravorty, Can the Subaltern Speak? (anche nella versione italiana).

55. Iuliano, Fiorenzo, Altri mondi, altre parole. Gayatri Chakravorty Spivak tra decostruzione e impegno militante, Verona, Ombre Corte, 2012, p. 139. 
parametri epistemologici con cui dobbiamo confrontarci. La considerazione dei subalterni non può, in questo senso, non tener conto e confrontarsi con la svolta epocale - "le grand partage" - con cui "l'etnologia abbatte la grande frontiera tra 'loro' e 'noi"'56 superando il modello vigente fin da Durkheim, secondo il quale esiste una barriera rigida e invalicabile fra il soggetto osservante e l'oggetto dell'osservazione scientifica. In pratica il rinnovamento auspicato da Said si è avverato e l'etnologia è costretta, oltre che a rivedere i propri protocolli, ad abbandonare le ricerche in terre lontane per confrontarsi, ad esempio, con flussi migratori entro $\mathrm{i}$ confini nazionali. Come classificare un migrante, come organizzare la comunicazione pubblica intorno ai sans-papiers? Qui si approda all'attualità sull'onda delle suggestioni di Marramao, il quale con Passaggio a Occidente ${ }^{57}$ ci presenta la rassegna finora più ricca e meglio argomentata di opzioni entro le quali troveranno una nuova collocazione, qualunque sia l'evoluzione politica dei prossimi anni a livello globale, le nozioni di subalterno e, quella correlata, di sud.

La conclusione aperta di questa breve escursione in quello che oggi intendiamo per mondo-sud vorrebbe ora poter fare a meno della scrittura per riprendere il concetto che Homi Bhabha rintraccia fra le righe delle osservazioni di Benjamin sul concetto di traduzione: nel corso di una traduzione, vi è un attimo in cui si manifesta l'elemento di resistenza nel processo di trasformazione, "ciò che in una traduzione non è a sua volta traducibile" 58 . "La cultura migrante dello spazio inter-medio, lo spazio dei minoritari non fa che mettere in scena, drammatizzare l'agire di questa intraducibilità della cultura $[. .$.$] ritrovando il$ processo ambivalente di scissione e ibridità che caratterizza l'identificazione della differenza con la cultura" ${ }^{59}$. Solo la capacità di mettere in atto l'accoglienza auspicata da Derrida come forma di dialogo interculturale offre dunque una prospettiva credibile per il mondo-sud.

Mauro PALA

Università di Cagliari

56. Clemente, Pietro, Mugnaini, Fabio (a cura di), Oltre il folklore. Tradizioni popolari e antropologia nella società contemporanea, Roma, Carocci, 2015, p. 43.

57. Marramao, Giacomo, Passaggio a Occidente. Filosofia della globalizzazione, Torino, Bollati Boringhieri, 2009 [2003].

58. Benjamin, Walter, "Il compito del traduttore", in iD., Angelus Novus, Torino, Einaudi, 1962, p. 45. Cit. in BнAвHA, Homi, I luoghi della cultura, Roma, Meltemi, 2001, p. 311.

59. Внавна, Homi, I luoghi della cultura, cit., p. 311. 
